DRAFT VERSION MARCH 31, 2020

Preprint typeset using $\mathrm{LAT}_{\mathrm{E}} \mathrm{X}$ style AASTeX6 v. 1.0

\title{
ALMA 50-PARSEC-RESOLUTION IMAGING OF JET-ISM INTERACTION IN THE LENSED QUASAR MG J0414+0534
}

\author{
KaIki TARo InOUE \\ Faculty of Science and Engineering, Kindai University, Higashi-Osaka, 577-8502, Japan \\ SATOKI Matsushita \\ Institute of Astronomy and Astrophysics, Academia Sinica, \\ 11F of Astronomy-Mathematics Building, AS/NTU, No.1, Sec.4, Roosevelt Rd., Taipei 10617, Taiwan, R.O.C. \\ Kouichiro NAKANISHI \\ National Astronomical Observatory of Japan, Mitaka, Tokyo 181-8588, Japan, \\ The Graduate University for Advanced Studies, SOKENDAI, Mitaka, Tokyo 181-8588, Japan \\ TAKeO MinezaKi \\ Institute of Astronomy, School of Science, University of Tokyo, Mitaka, Tokyo 181-0015, Japan
}

\begin{abstract}
We report our high-resolution (0!'03-0.'07) Atacama Large Millimeter/submillimeter Array (ALMA) imaging of the quadruply lensed radio-loud quasar MGJ0414+0534 at redshift $z=2.639$ in the continuum and the broad $\mathrm{CO}(11-10)$ line at $\sim 340 \mathrm{GHz}$. With the help of strong lensing magnification and ALMA's high resolution, we succeeded in resolving the jet/dust and $\mathrm{CO}$ gas in the quasar host galaxy both extending up to $\sim 1 \mathrm{kpc}$, with a resolution of $\sim 50 \mathrm{pc}$ for the first time. Both the continuum emission and the $\mathrm{CO}(11-10)$ line have a similar bimodal structure aligned with the quasar jets $(\sim 200 \mathrm{pc})$ observed by Very Long Baseline Interferometry (VLBI) at $5 \mathrm{GHz}$ and $8.4 \mathrm{GHz}$. The $\mathrm{CO}$ gas in the vicinity of both the eastern and western jet components at the location of $\sim 80 \mathrm{pc}$ from the quasar core are moving at high velocities, up to $\pm 600 \mathrm{~km} \mathrm{~s}^{-1}$ relative to the core. The observed features show clear evidence of strong interaction between the jets and interstellar medium (ISM). High temperature and high-density environments in the ISM of the quasar host galaxy, as suggested from CO line ratios, also support this result. The small scale of the jets, the jet-ISM interaction, and the continuum spectral energy distribution of this source indicate that we are watching the infancy stage of quasar radio activity.
\end{abstract}

Keywords: quasars: general — gravitational lensing: strong

\section{INTRODUCTION}

Recently, numerous observational studies in interactions between radio jets from active galactic nuclei (AGNs) and interstellar medium (ISM) have been reported. However, spatially resolved observations that can clearly see the jet-ISM interaction are still limited to a few objects. One of the nearest $(7.1 \mathrm{Mpc})$ Seyfert 2 galaxies, M51, shows both diffuse (CO) and dense (HCN) molecular gas along the radio jet, with those velocity gradients similar to that of an optical emission line on the jets. In addition, there is a density gradient with denser molecular gas closer to the jet. These observational results indicate the presence of the jet-entrained molecular gas (Matsushita et al. 2004, 2007, 2015). Relatively nearby $(47.9 \mathrm{Mpc})$ radio-loud Seyfert 2 galaxy
IC 5063 shows dense and warm molecular gas components with broad velocity width at the knots of radio jets, suggesting a jet-ISM interaction (Tadhunter et al. 2014; Morganti et al. 2015; Oosterloo et al. 2017). The above two examples clearly show the presence of interaction between radio jets and molecular gas. However, such interactions in early universe (i.e., high-z galaxies) have not been studied observationally. Such observations are important to study the evolution of AGNs and ISM.

Gravitationally lensed radio-loud quasars provide us excellent opportunity for investigating interactions between jet and ambient ISM in high-z galaxies. Among them, MG J0414+0534 (Hewitt et al. 1992), a quadruply lensed radio-loud quasar at redshift $z_{S}=2.639$ (Lawrence et al. 1995) is one the most promising targets due to its large magnification and spatially resolved 
small-scale $(\lesssim 1 \mathrm{kpc})$ radio jet components, observed at $5 \mathrm{GHz}$ (Trotter et al. 2000) and $8.4 \mathrm{GHz}$ (Ros et al. 2000).

In our Cycle 2 data (Inoue et al. 2017), we serendipitously discovered a broad $\mathrm{CO}(11-10)$ emission line in MG J0414+0534 (see also Stacey \& McKean 2018). Such a highly-excited CO line suggests the presence of molecular outflows. In ALMA Cycle 4, we proposed carrying out long baseline observations of the $\mathrm{CO}(11-10)$ line to probe the distribution and kinematics of molecular gas surrounding quasar jets on small scales.

In this letter, we present the results of our ALMA $\mathrm{Cy}_{-}$ cle 4 high angular resolution $\left(0{ }^{\prime \prime} 03-00^{\prime \prime} 05\right)$ observations (Project ID: 2016.1.00281.S, PI: S. Matsushita) combined with our Cycle 2 low angular resolution $\left(00^{\prime \prime} 1-00^{\prime \prime} 3\right)$ observations of the continuum emission and $\mathrm{CO}(11-10)$ line of MG J0414+0534 at $0.88 \mathrm{~mm}$. In what follows, we adopt a Planck 2018 cosmology with matter density $\Omega_{m, 0}=0.315$, energy density of cosmological constant $\Omega_{\Lambda, 0}=0.685$, and Hubble constant $H_{0}=67.4 \mathrm{~km} \mathrm{~s}^{-1}$.

\section{OBSERVATION AND REDUCTION}

Our ALMA Cycle 4 observations were carried out on 2017 November 1, 8, 10, and 11. The numbers of antennas used in the observations were $49,48,45$, and 49 , and the total on-source integrating times were $38.72,39.00$, 40.65 , and 38.88 minutes, respectively. The maximum and minimum baselines were $13.8944 \mathrm{~km}$ and $113 \mathrm{~m}$, respectively. The phase center was $\alpha=04^{\mathrm{h}} 14^{\mathrm{m}} 37^{\mathrm{s}} .7686$, $\delta=+05^{\circ} 34^{\prime} 42^{\prime \prime} \cdot 352$ (J2000). The ALMA correlator was configured to have four spectral windows centered at $335.370,337.120,347.182$, and $348.995 \mathrm{GHz}$, with a bandwidth $1.875 \mathrm{GHz}$ (frequency domain mode), to cover the $\mathrm{CO}(11-10)$ line $(1267.014486 \mathrm{GHz}$ at $z=0$, or $348.176556 \mathrm{GHz}$ at $z=2.639)$. The channel width was $0.976563 \mathrm{MHz}$ and the total channel number was 1920 for each window.

The calibration and imaging of the data were carried out using the Common Astronomy Software Application package (CASA; McMullin et al. 2007) version 5.1. In order to improve phase coherence, we carried out phaseonly self-calibration for our previous Cycle 2 (Inoue et al. 2017) and new Cycle 4 data, separately. In each iteration process, we selected continuum channels and the four spectral windows were combined to have a sufficient signal-to-noise ratio $(\mathrm{S} / \mathrm{N} \geq 3.0)$ for each gain solution.

After applying obtained calibration solutions to the whole data (continuum and lines), we carried out imaging with a natural weighting and further flagged some bad antennas by visual inspection of visibilities. After phaseonly self-calibration, our Cycle 2 data were significantly improved. The 'dynamic range,' defined as peak intensity per root-mean-square (rms) error, increased from $\sim 120$ to $\sim 250$. Applying phase-only self-calibration to our Cycle 4 data turned out to be difficult due to insufficient dynamic range in the original data. However, by reducing the condition for rejecting solutions for a group of three antennas to $\mathrm{S} / \mathrm{N}>2.0$, we obtained a dynamic range of 140 , which was improved by $\sim 20 \%$ compared to the original value.

After phase-only self-calibration, we combined the continuum data with weights inversely proportional to the variance of errors. In order to assess possible systematic differences in amplitude of visibilities, we compared both continuum data with a common $u v$-range between 140 and $1400 \mathrm{~m}$. It turned out that the difference is below $\sim 10 \%$, which is comparable to the typical error value in ALMA observations. We also subtracted the continuum emission from the line channels for both the Cycles 2 and 4 data in the $u v$ plane and combined them with weights inversely proportional to the variance of errors. The channel maps of the lensed CO(11-10) emission were generated with a width of $146 \mathrm{MHz}\left(125.6 \mathrm{~km} \mathrm{~s}^{-1}\right)$ per channel.

\section{RESULTS}

\subsection{Lensed images and line spectrum}

Fig. 1 shows the continuum image obtained from the Cycle 4 data and the integrated intensity of the $\mathrm{CO}(11-$ 10) line emission obtained from the combined Cycles 2 and 4 data. The continuum and CO images were obtained with a Briggs (robust $=0.5$ ) and a natural weightings, respectively. The achieved angular resolutions are $00^{\prime \prime} 03-0$. ".04 and $\sim 00^{\prime \prime} 07$, respectively. They show bright quadruply lensed spots A1, A2, B, and C. The continuum map shows some arcs on A1, A2, and B. The CO map shows that A1 consists of several spots, suggesting a more complex source structure than that of the continuum. The integrated flux and luminosity of the $\mathrm{CO}(11-$ 10) line are $6.3 \pm 0.6 \mathrm{Jy} \cdot \mathrm{km} / \mathrm{s}$ and $(1.1 \pm 0.1) \mu^{-1} \times 10^{9} \mathrm{~L}_{\odot}$, where $\mu$ is the total magnification. ${ }^{1}$

Fig. 2 shows the line spectrum of $\mathrm{CO}(11-10)$ obtained from the combined Cycles 2 and 4 data. We smoothed the cube data using a gaussian $u v$ tapering that yields beam sizes and a position angle of $\sim\left(2^{\prime \prime} \times 1^{\prime \prime}, 16^{\circ}\right)$ at each velocity bin, and only used the areas of the components $\mathrm{A} 1+\mathrm{A} 2$ and $\mathrm{B}$ with their integrated intensity $>3 \sigma$ in the lensed plane (the $\mathrm{CO}(11-10)$ spectrum is comparable to the $\mathrm{CO}(3-2)$ spectrum in Barvainis et al. 1998, see also Sect. 4). It shows a broad line width of full width at zero intensity $(\mathrm{FWZI})=1900 \mathrm{~km} \mathrm{~s}^{-1}$ and $\mathrm{FWHM}=1170 \pm$ $180 \mathrm{~km} \mathrm{~s}^{-1}$ obtained from a Gaussian fit. The dip at $\sim 140 \mathrm{~km} \mathrm{~s}^{-1}$ is due to missing channels in our Cycle 2 data taken in time domain mode.

1 The magnification factor for the mid-infrared emission is expected to be $\mu \sim 40$ (Takahashi \& Inoue 2014). 

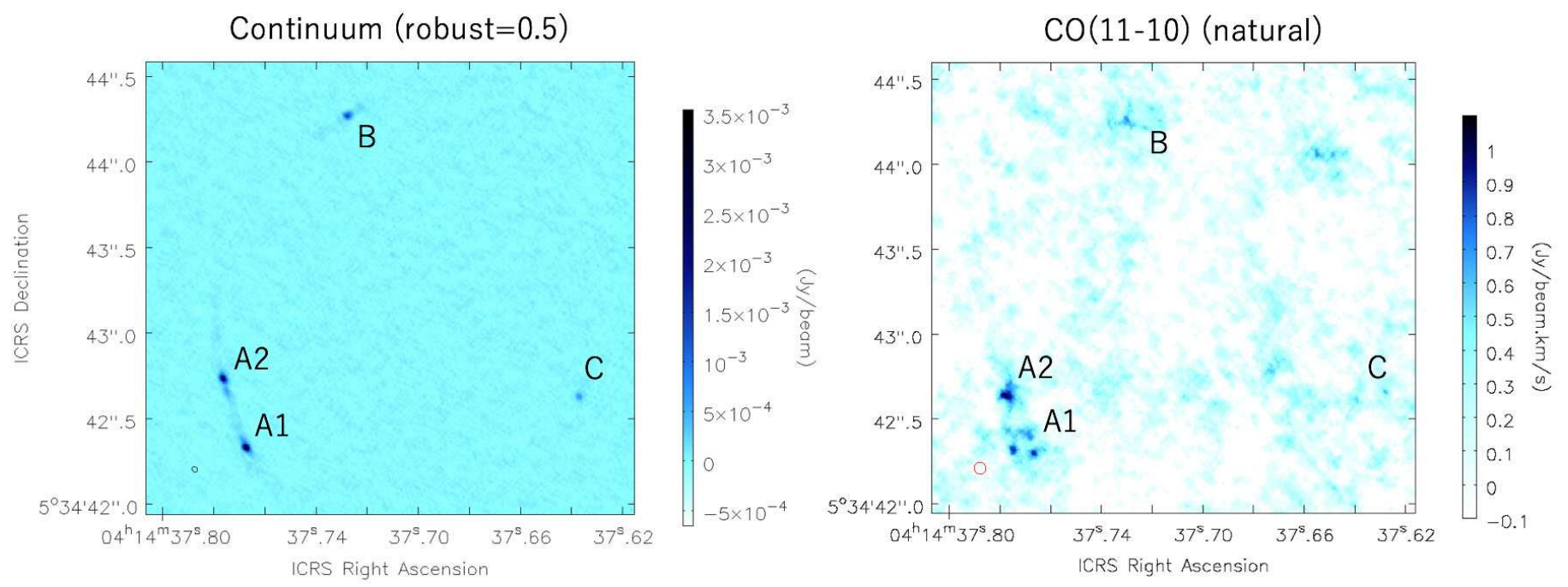

Figure 1. ALMA $0.88 \mathrm{~mm}$ (Band 7, 340 GHz) continuum and $\mathrm{CO}(11-10)$ images of MG J0414+0534. The Briggs weighting (robust $=0.5$ ) continuum image (left) was obtained from the Cycle 4 data, and the natural weighting integrated intensity image of the $\mathrm{CO}(11-10)$ (right) was obtained from the combined Cycles 2 and 4 data. The beam sizes and the position angles measured East of North, which are shown at the bottom left corner of each panel are $\left(0^{\prime \prime} 036 \times 0^{\prime \prime} \cdot 029,40.5\right)$ (left) and $\left(0^{\prime \prime} 072 \times 0^{\prime \prime} .068,18^{\circ} .2\right)$ (right). The rms noises and the total fluxes of lensed images are $(32 \mu \mathrm{Jy} /$ beam, $16 \pm 2 \mathrm{mJy})$ (left) and $(0.19 \mathrm{mJy} /$ beam $/$ channel, $6.3 \pm 0.6 \mathrm{Jy} \cdot \mathrm{km} / \mathrm{s})$ (right). For making the $\mathrm{CO}(11-10)$ image, only signals with $\gtrsim 1 \sigma$ in each channel map were used.

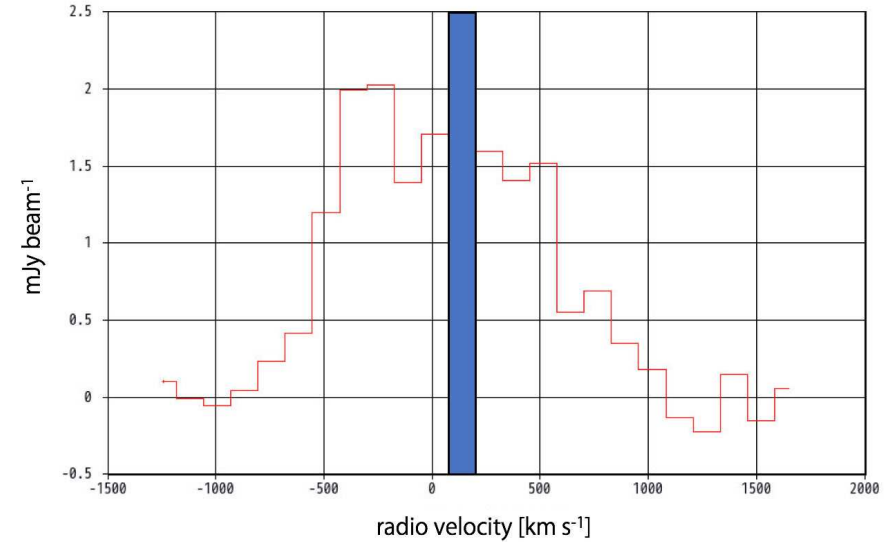

Figure 2. The spectrum profile of the continuum subtracted $\mathrm{CO}(11-10)$ line emission for $\mathrm{A} 1, \mathrm{~A} 2$, and $\mathrm{B}$ over the full bandwidth (red). The blue rectangular box at $\sim 140 \mathrm{~km} \mathrm{~s}^{-1}$ corresponds to missing channels in the $\mathrm{Cy}$ cle 2 data. The velocity offsets are relative to a redshift of 2.639. To obtain the spectrum, we smoothed the cube data to have the same angular resolution as in the $\mathrm{CO}(3-$ 2) data (Barvainis et al. 1998).

\subsection{Lens modeling and de-lensing}

To model the observed images, we first made an unperturbed 'background' model without using our ALMA data. It consists of a singular isothermal ellipsoid for a lensing galaxy $\mathrm{G}$ plus a cored isothermal sphere for a possible companion galaxy, object X (see Falco et al. 1997), and an external shear. For fitting relative positions of the lensed images, we used the measured Hubble Space Telescope(HST) Wide Field and Planetary Camera/Wide Field and Planetary Camera 2 (WFPC/WFPC2) positions and centroids of lensing galaxies, $\mathrm{G}$ and $\mathrm{X}$ in the CASTLES data archive ${ }^{2}$. For fitting flux ratios of lensed images, we used the mid-infrared (MIR) flux ratios of A2/A1 and B/A1 (Minezaki et al. 2009; MacLeod et al. 2013). The Very Long Baseline Interferometry (VLBI) positions of the lensed possible core components at $5 \mathrm{GHz}$ (Trotter et al. 2000) were fit to the corresponding HST positions. The residual errors in the relative positions are $<00^{\prime \prime} 003$, which are less than the errors in the HST relative positions.

In order to improve our model, we took into account the lensing effects from possible substructures (subhalos and line-of-sight halos/voids) using our ALMA Cycles 2 and 4 combined continuum image (not shown here). We obtained a best-fit model by minimizing the differences between the de-lensed images, while the astrometric shifts and magnification perturbations were constrained to fit the optical/near-infrared (NIR) and MIR data (see Inoue et al. 2016, 2020, for detail). After fitting, the positions of possible core components $p$ and $q$, jet components $r$ and $s$ at $5 \mathrm{GHz}$ (see Trotter et al. 2000, for the definition) and the optical/IR data were fit to the observed data.

Using the best-fit lensing model, we reconstructed de-

2 http://www.cfa.harvard.edu/castles/ 
lensed images of the continuum and $\mathrm{CO}(11-10)$ line from a linear combination of three de-lensed images of A1, A2 and $\mathrm{B}$. Image $\mathrm{C}$ was not taken into account as the angular resolution of de-lensed image $\mathrm{C}$ was too low. From the de-lensed images, we found that the total magnification factors for the continuum and $\mathrm{CO}(11-10)$ are $37 \pm 4$ and $28 \pm 3$, respectively.

\subsection{Continuum}

Fig. 3(a) shows the de-lensed continuum image of the Cycle 4 data $(\text { robust }=0.5)^{3}$. It shows a very bright compact emission at the position of the core component $q$. Moreover, the best-fit source position of the MIR emission (denoted by a green cross in Fig. 3) seems to coincide with that of $q$. Though not conclusive, our result suggests that $q$ corresponds to quasar circumnuclear dust emission or synchrotron radiation from the quasar core, whereas another possible core component $p$ corresponds to emission from the quasar jet near the core.

In order to further probe the structure near the core, we attempted to reduce the side effect due to the 'side lobes' of $p$ and $q$ in the source plane as they are extremely bright. To do so, we subtracted off the best-fit Gaussians at the lensed $p$ and $q$ images. The Gaussian point spread function (PSF) flux ratios were chosen to be consistent with the VLBI flux ratios of $p$ and $q$ at $5 \mathrm{GHz}$ (Trotter et al. 2000; Inoue et al. 2020). The absolute amplitudes of the lensed $p$ and $q$ at A2 were chosen as free parameters as they have a relatively large flux and separation. The absolute amplitudes were determined appropriately through visual inspection of the smoothness of the image in which the fitted Gaussian PSFs are subtracted off. The achieved angular resolution near intensity peak in the source plane was found to be $\sim 0^{\prime \prime} 007^{4}$, which corresponds to $\sim 50 \mathrm{pc}$ at $z_{S}=2.639$.

Fig. 3(b) shows the de-lensed continuum image of Cycle 4 data (robust $=0.5)$ in which $p+q$ are subtracted off. In the image, one can clearly see a bimodal structure along jet components. The positions of local peak intensity in the bimodal structure are slightly shifted toward $q$ from the best-fit positions of the other jet components $s$ and $r$. The origin and the emission mechanism of the bimodal structure is considered to be either synchrotron emission from the quasar jets or dust emission, or both. The size of the de-lensed continuum emitting region is $0.5-1 \mathrm{kpc}$. We can also see a filamentary structure that looks like a spiral-arm or a stream in the southeast of $q$, which implies clumpy structure in the dust. The length of the 'arm' (or 'stream') is $\sim 400 \mathrm{pc}$, which is approximately a half of

3 We also made a de-lensed image using our Cycles 2 and 4 combined data. However, the angular resolution of the de-lensed image was slightly worse than the image in Fig. 3.

4 The resolution can be evaluated from the beam size of the Cycle 4 continuum image and the magnification factors in the vicinity of intensity peaks dusty substructures observed in luminous submillimeter galaxies (Hodge et al. 2019).

\section{4. $C O(11-10)$}

Fig. 3(c) and (d) show the de-lensed CO(11-10) integrated intensity and intensity weighted velocity field maps. In order to increase the $\mathrm{S} / \mathrm{N}$, we only picked up pixels with $\mathrm{S} / \mathrm{N}>2$ within the velocity range of $-700<V<800 \mathrm{~km} / \mathrm{s}$ and combined them. For the velocity definition, we assumed that the core $q$ has a zero velocity: the center velocity was shifted by $-75 \mathrm{kms}^{-1}$ from $z_{S}=2.639$, meaning that the rest frame is at the redshift of the $\mathrm{CO}$ emitting core, $z_{\mathrm{CO}}=2.6381$. In the de-lensed integrated intensity image (Fig. 3c), no strong core component can be seen, which is contrary to the continuum image that shows a strongest peak in there. Instead, we can see a bimodal structure along jet components in a similar way as is the continuum image with PSFs subtracted. The positions of the local peak intensity appear to roughly coincide with the brightest regions in the PSFs subtracted continuum image; they reside in the vicinity of jet components $s$ and $r$, though slightly shifted toward $q$. In the velocity field map (Fig. 3d), one can see a velocity gradient of $-2 \mathrm{~km} / \mathrm{spc}^{-1}$ along a section that connects $q$ and $r$. However, it turned out that no such velocity gradient can be seen along a section that connects $q$ and $s$. Such an asymmetric feature cannot be explained by a simple circular rotating disk or a spheroid.

In order to inspect possible interaction between jet and gas, we made a position-velocity (PV) diagram (Fig. 4) on a section that best fits the positions of the three jet components $s, p$ and $r$ and the core component $q$ using $\chi^{2}$ minimization (Fig. 3d). Data at $\sim 140 \mathrm{~km} \mathrm{~s}^{-1}$ were omitted due to missing channels in our Cycle 2 'time domain mode' observations. One can see that the brightest spot in the vicinity of the eastern jet component $s$ has velocities up to $\pm 600 \mathrm{~km} \mathrm{~s}^{-1}$. In addition, the CO gas in the vicinity of the western jet component $r$ is moving at velocities from $-300 \mathrm{kms}^{-1}$ to $600 \mathrm{~km} \mathrm{~s}^{-1}$. Both the $s$ and $r$ components are located at a distance of $\sim 80 \mathrm{pc}$ from the quasar core component $q$. Even some components are moving at velocities from $-400 \mathrm{~km} \mathrm{~s}^{-1}$ to $400 \mathrm{~km} \mathrm{~s}^{-1}$ at the outer side of $s$ and from $-200 \mathrm{~km} \mathrm{~s}^{-1}$ to $600 \mathrm{~km} \mathrm{~s}^{-1}$ at the outer side of $r$, both with respect to $q$.

\section{DISCUSSION}

We detected a broad (FWZI $\sim 1900 \mathrm{kms}^{-1}$ and FWHM $\left.\sim 1170 \pm 180 \mathrm{~km} \mathrm{~s}^{-1}\right)$, high-J $(\mathrm{J}=11-10) \mathrm{CO}$ emission line from the lensed quasar MG J0414+0534. This line is much broader than the lower transition $\mathrm{CO}(3-$ 2) line with FWHM $=580 \mathrm{~km} \mathrm{~s}^{-1}$ (Barvainis et al. 1998). Since the $\mathrm{S} / \mathrm{N}$ of the $\mathrm{CO}(3-2)$ data is much worse than that of our $\mathrm{CO}(11-10)$ data, weak high-velocity compo- 

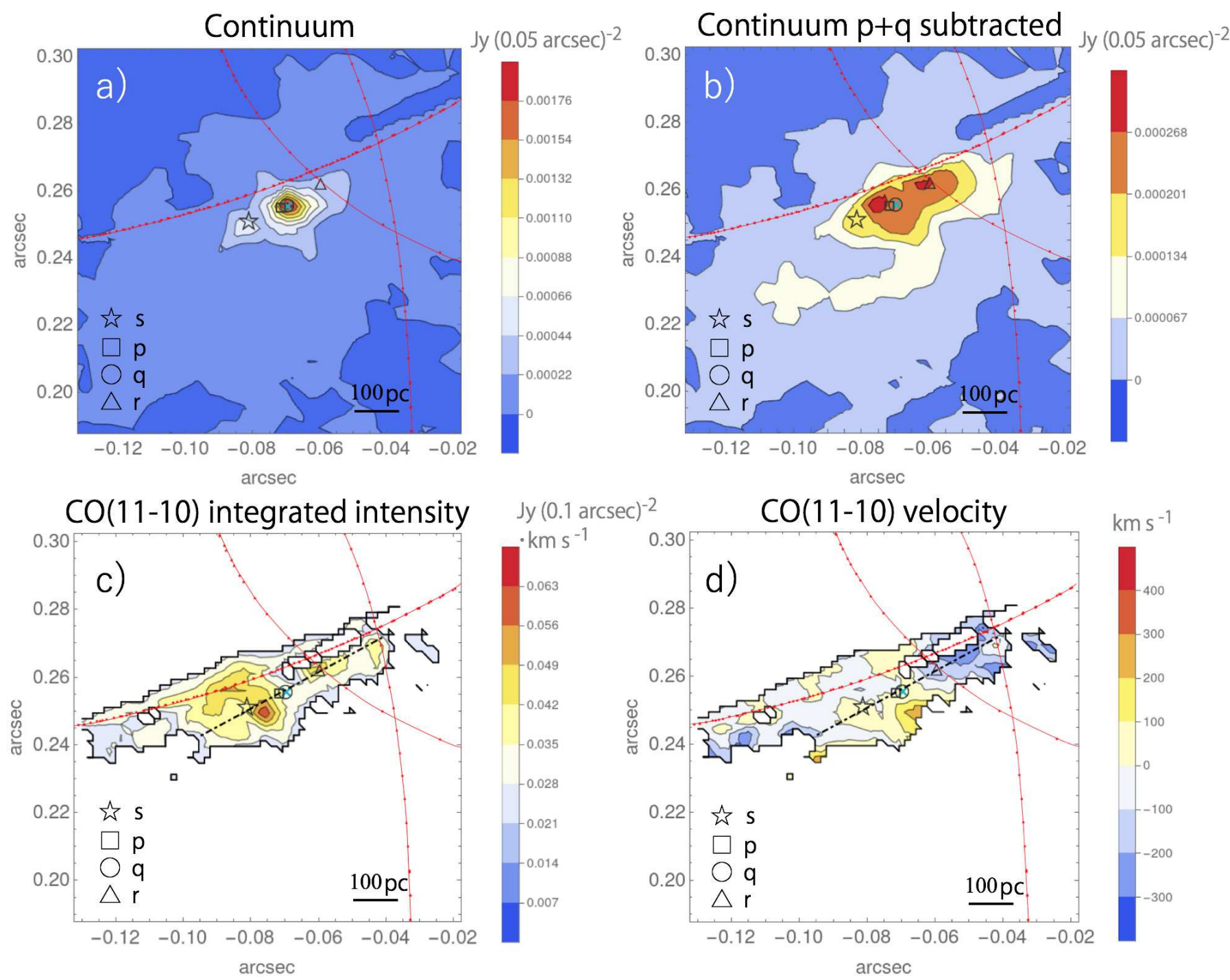

Figure 3. (a) De-lensed continuum image of the Cycle 4 data before subtraction of $p+q$, (b) de-lensed continuum image of the Cycle 4 data with $p+q$ subtracted, (c) de-lensed $\mathrm{CO}(11-10)$ integrated intensity over the full band width, and (d) de-lensed $\mathrm{CO}(11-10)$ intensity weighted velocity maps. In all the maps, $0^{\prime \prime} 01$ corresponds to a physical scale of $82 \mathrm{pc}$. The coordinates are in the FK5 frame at J2000.0 centered at the centroid of a lensing galaxy G. The spatial resolution of the continuum image is $\sim 50 \mathrm{pc}$ and that of the $\mathrm{CO}(11-10)$ image is $\sim 100 \mathrm{pc}$. The contours start from $5 \sigma$ for (a) and (b), $3 \sigma$ for (c). The spacings are $10 \sigma$ for (a), $3 \sigma$ for (b), $1 \sigma$ for (c) and $100 \mathrm{~km} \mathrm{~s}^{-1}$ for (d). For both of the $\mathrm{CO}(11-10)$ maps, pixels below the $3 \sigma$ in the integrated intensity map are masked out, and the limit is expressed as thick contours. The center velocity was shifted by $-75 \mathrm{~km} \mathrm{~s}^{-1}$ from $z_{S}=2.639$ to make the quasar core $q$ be at rest in the intensity weighted velocity map. The green cross in each panel corresponds to the best-fit position of the MIR emission from the quasar dust core. Red dots and fitted curves correspond to the caustics. The dashed-dotted line in the $\mathrm{CO}(11-10)$ maps shows a section for the position-velocity (PV) diagram in Fig. 4.

nents may have not been detected in the $\mathrm{CO}(3-2)$ data. Alternatively, the high-velocity components are dominated by highly excited $\mathrm{CO}$ and affected by the differential magnification effect (Serjeant 2012). Assuming that both the lines except for the high-velocity components are emitting from the same region, restricting the channels of $\mathrm{CO}(11-10)$ to have a line width of $6 \times 10^{2} \mathrm{~km} \mathrm{~s}^{-1}$, which is equivalent to the FWHM of the
$\mathrm{CO}(3-2)$ data, the $\mathrm{CO}(11-10) /(3-2)$ brightness temperature ratio is found to be 0.14 . Since the $\mathrm{CO}(1-0)$ line has not been detected, the lower limit of the $\mathrm{CO}(3-2) /(1-0)$ line luminosity ratio is estimated as 2.56 (Sharon et al. 2016), and that of the $\mathrm{CO}(3-2) /(1-0)$ brightness temperature ratio is estimated as 0.475 . Using RADEX (van der Tak et al. 2007), we found that a kinetic temperature of $\sim 2 \times 10^{2} \mathrm{~K}$ and a $\mathrm{H}_{2}$ number density of 


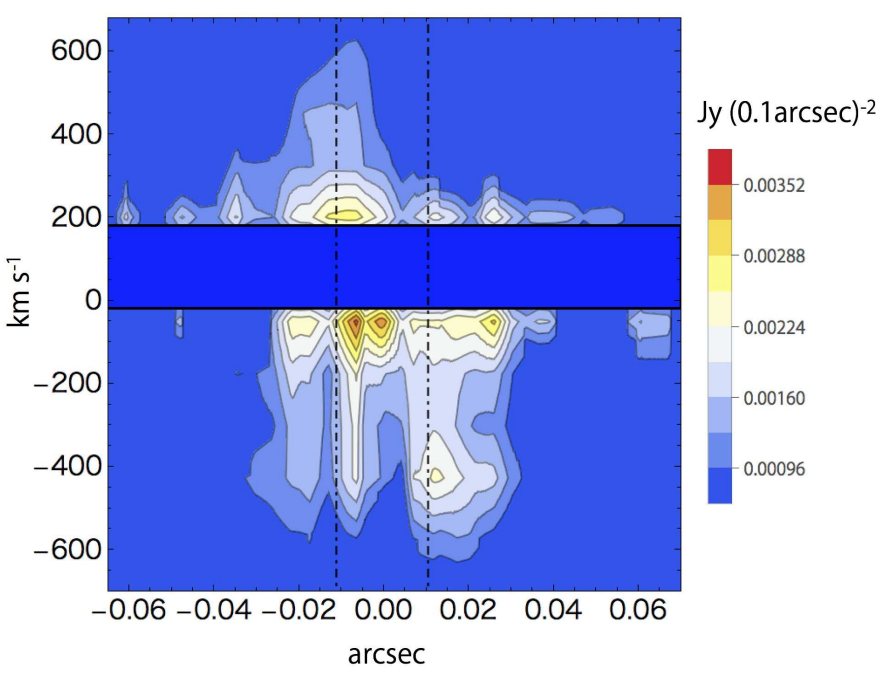

Figure 4. Position-velocity (PV) diagram of the delensed $\mathrm{CO}(11-10)$ data on a section that best fits the positions of all the components $p, q, r$, and $s$ (see Fig. 3(d)). The data around $\sim 140 \mathrm{kms}^{-1}$ are omitted due to misisng channels in our Cycle 2 observations (see also Fig. $2)$. The dot-dashed vertical lines show the position offsets of jet components $s$ (left line) and $r$ (right line) with respect to $q$ measured in the section. Pixels below the $3 \sigma$ in intensity are masked out. The contour spacing is $1 \sigma$. The zero-velocity point corresponds to the rest frame of the quasar core $q$ and the zero-position point corresponds to the position that is nearest to $q$ in the section.

$\sim 5 \times 10^{4} \mathrm{~cm}^{-3}$ are consistent with the brightness temperature ratios of CO. These facts indicate that the molecular gas in MG J0414+0534 is in high-temperature and high-density conditions.

Such conditions are likely due to the interaction between the jets and molecular gas. First, our ALMA data show a broad $\mathrm{CO}(11-10)$ line emission along the radio jet components. The positions of the bright $\mathrm{CO}(11-10)$ components coincide well with those of the bright radio jet components, and those $\mathrm{CO}(11-10)$ components have broad line widths (Sect. 3.4), which are very similar to what has been observed in IC 5063 (Morganti et al. 2015). Secondly, the $22 \mathrm{GHz}$ water maser lines are detected from this lensed quasar, with their velocities (the central velocities of two strongest line components are at $-278 \mathrm{kms}^{-1}$ and $+470 \mathrm{kms}^{-1}$ ) well within the broad $\mathrm{CO}(11-10)$ line width, and it is suggested that the water maser lines come from the interaction zone of the radio jet and the molecular gas (Impellizzeri et al. 2008; Castangia et al. 2011).

The high-temperature and high-density conditions are also consistent with the characteristics of the continuum radio emission. The spectral energy distribution (SED) of the continuum radio emission of MG J0414+0534 has a turnover around $0.4-0.5 \mathrm{GHz}$, and therefore MGJ0414+0534 is classified as a giga-peaked spectrum (GPS) source (Katz et al. 1997; Edwards \& Tingay 2004). The major cause of the SED turn-over in GPS sources is considered as either synchrotron selfabsorption or free-free absorption (see O'Dea 1998, for a review). Together with the small size ( $\lesssim 1 \mathrm{kpc})$ of their radio emitting regions, it implies that radio jets in GPS sources are still interacting with the ISM in the host galaxies (O'Dea \& Baum 1997). The compactness of the continuum emitting region of 500-1000 pc (Sect. 3.3) and the molecular gas - jet interaction we discussed above in MG J0414+0534 are well consistent with the characteristics of GPS sources.

It is suggested that GPS sources are in the young stage of the evolution of radio galaxies on the grounds of their small sizes (O'Dea 1998) as seen in MG J0414+0534. On the other hand, GPS sources might be so frustrated that the expansions of jets are regulated by the dense ISM, and the condition could make the sizes of the GPS sources relatively small with respect to their old ages (O'Dea \& Baum 1997). Nonetheless, the ages of GPS sources are supposed to be as young as $\lesssim 1 \times 10^{5}$ years even under the frustrated conditions. Recent numerical simulations of jet-ISM interaction show that even though dense ISM is surrounding radio sources, jets can expand to $\sim 1000 \mathrm{pc}$ within the order of $10^{4}$ years (Wagner \& Bicknell 2011; Wagner et al. 2012). On the other hand, intermittent radio jet activity can be a counterargument against young age of GPS sources. However, there is no observational evidence that supports intermit-

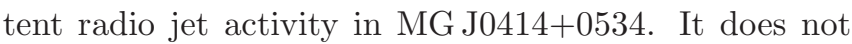
have any extended radio emission, and also most of GPS sources do not have any extended radio emission ( $O^{\prime} D e a$ 1998).

Based on the evidence above, we conclude that MG J0414+0534 is possibly in the early stage of quasar radio activity. We expect that the radio jets recently emitted from the AGN is currently drilling the ISM inside the host galaxy. The interaction between the radio jets and the ISM in the quasar host galaxy causes the highly excited broad $\mathrm{CO}(11-10)$ line and the water maser lines.

Recent observations toward AGNs and quasars detected highly excited CO lines (e.g., Hailey-Dunsheath et al. 2012). The jet-ISM interaction may be responsible for heating the CO gas. Our observations would provide an important insight on a possible excitation mechanism for high-J CO lines.

KTI would like to thank Eiji Akiyama, Misato Fukagawa and Fumi Egusa for their support on data 
reduction, and acknowledges support from NAOJ ALMA Scientific Grant Number 2018-07A and JSPS KAKENHI Grant Number 17H02868. SM is supported by the Ministry of Science and Technology (MoST) of Taiwan, MoST 103-2112-M-001-032MY3, 106-2112-M-001-011, and 107-2119-M-001-020. KN is supported by JSPS KAKENHI Grant Number 19K03937. This Letter makes use of the following ALMA data: ADS/JAO.ALMA\#2013.1.01110.S., 2016.1.00281.S. ALMA is a partnership of ESO (representing its member states), NSF (USA) and NINS (Japan), together with NRC (Canada) and NSC and ASIAA (Taiwan), and KASI (Republic of Korea) in cooperation with the Republic of Chile. The Joint ALMA Observatory is operated by ESO, AUI/NRAO, and NAOJ.

\section{REFERENCES}

Barvainis, R., Alloin, D., Guilloteau, S., \& Antonucci, R. 1998, ApJL, 492, L13

Castangia, P., Impellizzeri, C. M. V., McKean, J. P., et al. 2011, A\&A, 529, A150

Edwards, P. G., \& Tingay, S. J. 2004, A\&A, 424, 91

Falco, E. E., Lehar, J., \& Shapiro, I. I. 1997, ApJ, 113, 540

Hailey-Dunsheath, S., Sturm, E., Fischer, J., et al. 2012, ApJ, 755,57

Hewitt, J. N., Turner, E. L., Lawrence, C. R., Schneider, D. P., \& Brody, J. P. 1992, ApJ, 104, 968

Hodge, J. A., Smail, I., Walter, F., et al. 2019, ApJ, 876, 130

Impellizzeri, C. M. V., McKean, J. P., Castangia, P., et al. 2008, Nature, 456, 927

Inoue, K. T., Matsushita, S., Minezaki, T., \& Chiba, M. 2017, ApJL, 835, L23
Inoue, K. T., Minezaki, T., Matsushita, S., \& Chiba, M. 2016, MNRAS, 457, 2936

Inoue, K. T., et al. 2020, in preparation

Katz, C. A., Moore, C. B., \& Hewitt, J. N. 1997, ApJ, 475, 512

Lawrence, C. R., Elston, R., Januzzi, B. T., \& Turner, E. L. 1995, AJ, 110, 2570

MacLeod, C. L., Jones, R., Agol, E., \& Kochanek, C. S. 2013, ApJ, 773, 35

Matsushita, S., Muller, S., \& Lim, J. 2007, A\&A, 468, L49

Matsushita, S., Trung, D.-V., Boone, F., et al. 2015, ApJ, 799, 26

Matsushita, S., Sakamoto, K., Kuo, C.-Y., et al. 2004, ApJL, 616, L55

McMullin, J. P., Waters, B., Schiebel, D., Young, W., \& Golap, K. 2007, in Astronomical Society of the Pacific Conference Series, Vol. 376, Astronomical Data Analysis Software and Systems XVI, ed. R. A. Shaw, F. Hill, \& D. J. Bell, 127

Minezaki, T., Chiba, M., Kashikawa, N., Inoue, K. T., \& Kataza, H. 2009, ApJ, 697, 610

Morganti, R., Oosterloo, T., Oonk, J. B. R., Frieswijk, W., \&

Tadhunter, C. 2015, A\&A, 580, A1

O'Dea, C. P. 1998, PASP, 110, 493

O'Dea, C. P., \& Baum, S. A. 1997, AJ, 113, 148

Oosterloo, T., Raymond Oonk, J. B., Morganti, R., et al. 2017, A\&A, 608, A38

Ros, E., Guirado, J. C., Marcaide, J. M., et al. 2000, A\&A, 362, 845

Serjeant, S. 2012, MNRAS, 424, 2429

Sharon, C. E., Riechers, D. A., Hodge, J., et al. 2016, ApJ, 827, 18

Stacey, H. R., \& McKean, J. P. 2018, MNRAS, 481, L40

Tadhunter, C., Morganti, R., Rose, M., Oonk, J. B. R., \& Oosterloo, T. 2014, Nature, 511, 440

Takahashi, R., \& Inoue, K. T. 2014, MNRAS, 440, 870

Trotter, C. S., Winn, J. N., \& Hewitt, J. N. 2000, ApJ, 535, 671

van der Tak, F. F. S., Black, J. H., Schöier, F. L., Jansen, D. J., \& van Dishoeck, E. F. 2007, A\&A, 468, 627

Wagner, A. Y., \& Bicknell, G. V. 2011, ApJ, 728, 29

Wagner, A. Y., Bicknell, G. V., \& Umemura, M. 2012, ApJ, 757, 136 